\title{
Inhibitory potentials of Cymbopogon citratus oil against aluminium-induced behavioral deficits and neuropathology in rats
}

\author{
Gbadamosi Ismail Temitayo ${ }^{1}$, Bamisi Olawande ${ }^{1}$, Yawson Olushola Emmanuel ${ }^{1,2}$, \\ Arogundade Tolulope Timothy ${ }^{1,2}$, Ogunrinola Kehinde ${ }^{1}$, Lewu Folashade Susan ${ }^{1}$, Lambe Ezra ${ }^{1}$, \\ Olajide Olayemi Joseph ${ }^{1,3}$ \\ ${ }^{1}$ Division of Neurobiology, Department of Anatomy, Faculty of Basic Medical Sciences, College of Health Sciences, University of Ilorin, Ilorin, ${ }^{2}$ Division \\ of Neurobiology, Department of Anatomy, Faculty of Basic Medical Sciences, Adeleke University, Ede, Nigeria, ${ }^{3}$ Center for Studies in Behavioral \\ Neurobiology, Department of Psychology, Concordia University Montreal, Montreal, Quebec, Canada
}

Abstract: Cymbopogon citratus is a tropical phytomedicinal plant that is widely known for its hypoglycemic, hypolipidemic, anxiolytic, sedative, antioxidative and anti-inflammatory properties. In this study, we have examined the neuroprotective effects of the essential oil (ESO) of Cymbopogon citratus, following aluminum chloride $\left(\mathrm{AlCl}_{3}\right)$-induced neurotoxicity within the cerebellum of Wistar rats. A total of 40 adult male Wistar rats were assigned into five groups and treated orally as follows: A-phosphate-buffered saline ( $1 \mathrm{ml}$ daily for 15 days); B-ESO $\left(50 \mathrm{mg} / \mathrm{kg}\right.$ daily for 15 days); $\mathrm{C}-\mathrm{AlCl}_{3}$ (100 mg/kg daily for 15 days); $\mathrm{D}-\mathrm{AlCl}_{3}$ then $\mathrm{ESO}(100 \mathrm{mg} / \mathrm{kg} \mathrm{AlCl}$ daily for 15 days followed by $50 \mathrm{mg} / \mathrm{kg}$ ESO daily for subsequent 15 days); EESO then $\mathrm{AlCl}_{3}(50 \mathrm{mg} / \mathrm{kg}$ ESO daily for 15 days followed by $100 \mathrm{mg} / \mathrm{kg} \mathrm{AlCl}$ daily for following 15 days). To address our questions, we observed the locomotion and exploratory behavior of the rats in the open field apparatus and subsequently evaluated cerebellar oxidative redox parameters, neural bioenergetics, acetylcholinesterase levels, transferrin receptor protein, and total protein profiles by biochemical assays. Furthermore, we investigated cerebellar histomorphology and Nissl profile by H\&E and Cresyl violet Nissl staining procedures. ESO treatment markedly attenuated deficits in exploratory activities and rearing behavior following $\mathrm{AlCl}_{3}$ toxicity, indicating its anxiolytic potentials. Additionally, $\mathrm{AlCl}_{3}$ evokedincrease in malondialdehyde and nitric oxide levels, as well as repressed cerebellar catalase, glutathione peroxidase, and superoxide dismutase profiles were normalised to baseline levels by ESO treatment. Treatment with ESO, ergo, exhibits substantial neuroprotective and modulatory potentials in response to $\mathrm{AlCl}_{3}$ toxicity.

Key words: Cerebellum, Cymbopogon, Inflammation, Oxidative stress

Received April 23, 2020; Revised May 19, 2020; Accepted May 25, 2020

\section{Corresponding author:}

Gbadamosi Ismail Temitayo (i)

Division of Neurobiology, Department of Anatomy, Faculty of Basic Medical Sciences, College of Health Sciences, University of Ilorin, Ilorin, PMB 1515, Nigeria

E-mail: ismail.tayo@yahoo.com

\section{Introduction}

Alzheimer's disease (AD) is a complex, progressive, and chronic neurodegenerative condition that leads to learning, cognitive and behavioral impairment that results from neuronal and synaptic deficits in essential brain areas $[1,2]$. Neuritic plaques comprising $\beta$-amyloid (A $\beta$ ) peptide as well as intraneuronal neurofibrillary tangles composed of aggregated hyperphosphorylated microtubule-associated protein 
tau, extracellular amyloid are parts of the pathological observations associated with $\mathrm{AD}$ [3]. Most studies on AD focus on the cerebral cortex and hippocampus with undoubtedly established associated roles in learning, memory, and behavioral functions, consequently causing researchers to pay less attention to the cerebellum and other areas of the brain during the clinical onset as well as the progression of $\mathrm{AD}$ pathology. Despite the emergence of investigative studies on the cerebellum in AD pathology suggesting that degenerative changes within the cerebellum may be inclusive in both pathogenesis and symptomatic manifestation of $\mathrm{AD}$, there is still a paucity of comprehensive scientific evidence to substantiate the neuropathological observations occurring in the cerebellum of AD subjects $[4,5]$. Such scientific evidence is imperative for isolating potential therapeutic agents against neuronal loss in $\mathrm{AD}$ and preventing resulting behavioral deficits.

Several data have described the roles of aluminum chloride $\left(\mathrm{AlCl}_{3}\right)$ in the etiology of $\mathrm{AD}$. $\mathrm{Al}$, as an element is environmentally ubiquitous, its administration has been reported to induce neurological pathology via several routes, such as provoking dysfunction and activation of glial cells, which play an indispensable role in the regulation of the central nervous system homeostasis and neurodevelopment $[6,7]$. Other routes include the generation of oxidative stress, depletion of reduced glutathione, direct and indirect reductions in mitochondrial performance and functional integrity, and increasing the production of pro-inflammatory cytokines in both the brain and peripherally [8]. Behavioral, neuroanatomical, and neurochemical alterations that mimic $\mathrm{AD}$ are established characteristic pathological hallmarks of both acute and chronic exposure to $\mathrm{Al}$. Al promotes the formation and accumulation of insoluble $A \beta$ and hyper-phosphorylated tau [7]. To some extent, $\mathrm{Al}$ creates a deficit in cortical cholinergic neurotransmission, while it increases Fe-induced oxidative injury, as seen in $\mathrm{AD}$ [9]. The toxicity of $\mathrm{Al}$ to plants, aquatic life, and humans may share common mechanisms, including disruption of the inositol phosphate system and calcium dysregulation. [10, 11].

Cymbopogon citratus, also known as lemongrass, is a tropical phytomedicinal plant that has been widely explored for its therapeutic potentials. Studies have previously reported its hypoglycemic, hypolipidemic, anxiolytic, sedative, antioxidative, and anti-inflammatory effects [12-16]. In past years, the phenolic composition of lemongrass infusion was described to include: 3 -feroylquinic acid, neochlorogenic acid, chlorogenic acid, p-coumaric acid, carlinoside, isoschaftoside, isoorientin, cynaroside, veronicastroside, luteolin 7-O-neohesperidose, kurilensin A and cassiaoccidentalin B [17]. Flavonoids, such as luteolin, apigenin, glycosides, and tannins, strongly contributed to the antioxidant and anti-inflammatory properties of lemongrass [18]. The antiinflammatory and analgesic potential of lemongrass infusion has also been reported [19].

Biological effects of flavonoids imply their bioactivity and bioavailability, and their ability to effectively reach target tissues. Therefore, using behavioral, histochemical and biochemical approaches to investigate whether extracted essential oil (ESO) of lemongrass can modulate disrupted antioxidant enzymes, altered cholinergic neurotransmission, dysfunctional neuronal bioenergetics, induction of irregular protein synthesis and neuronal cell death in cerebelli of Wistar rats were the primary objectives of the present study. Here, we have characterised the effects of chronic $\mathrm{AlC}_{3}$ exposure on the cerebellum of Wistar rats at multiple levels of organisation and complexity, while investigating the preventive and therapeutic potential of ESO in vivo.

\section{Materials and Methods}

\section{Extraction of essential oil of Cymbopogon citratus}

The freeze-dried sample of lemongrass (200 g; obtained from a plantation at Oke-Ose area of Ilorin in October of 2016) was steam-distilled for 1.5 hours in a Clevenger-type apparatus, with $700 \mathrm{ml}$ of water at fixed temperature and extraction time based on the optimisation range to give a mixture of water/ESO. The ESO was cooled and separated from the water layer by using dichloromethane and dried in excess of anhydrous sodium sulfate. It was then stored in the dark at $4^{\circ} \mathrm{C}$. Salt of $\mathrm{AlCl}_{3}$ was dissolved in distilled water to produce $20 \mathrm{mg} / \mathrm{ml}$ of $\mathrm{AlCl}_{3}$ solution and adjusted to $\mathrm{pH}$ 7.4.

\section{Animal grouping and treatments}

A total of 40 male rats $(185 \pm 3 \mathrm{~g})$ were randomly assigned into five groups (A-E, $\mathrm{n}=8$ ) and treated orally as follows: Aphosphate-buffered saline (PBS) (1 ml daily for 15 days); BESO (50 mg/kg daily for 15 days); $\mathrm{C}-\mathrm{AlCl}_{3}(100 \mathrm{mg} / \mathrm{kg}$ daily for 15 days); $\mathrm{D}-\mathrm{AlCl}_{3}$ then $\mathrm{ESO}\left(100 \mathrm{mg} / \mathrm{kg} \mathrm{AlCl}_{3}\right.$ daily for 15 days followed by $50 \mathrm{mg} / \mathrm{kg}$ ESO daily for subsequent 15 days); E-ESO then $\mathrm{AlCl}_{3}(50 \mathrm{mg} / \mathrm{kg}$ ESO daily for 15 days followed by $100 \mathrm{mg} / \mathrm{kg} \mathrm{AlCl}_{3}$ daily for subsequent 15 days). 


\section{Open field test}

The experimental animals in this study were subjected to the open field behavioral paradigm 24 hours after the final administration. The open field apparatus was made from plywood measuring $100 \times 100 \mathrm{~cm}$ with walls $50 \mathrm{~cm}$ high. The floor was divided into square grids, each measuring $25 \mathrm{~cm}$ in length with a blue marker, and a center square of the same length was drawn with a red marker. During the test, the rats were picked by their tails and dropped in the center square and allowed to explore for 5 minutes while a video was being captured by a camera from above the apparatus. Five behaviors were scored from outcomes, and these include the number of lines crossed, center square entry, center square duration, rearing frequency, and stretch-attend posture. The number of lines crossed was the frequency with which the rats crossed one of the grid lines with all four paws. The center square entry was the frequency with which the rats crossed one of the red lines with all four paws into the central square while the center square duration was the total time spent in the center square. The rearing frequency was the number of times the animal stood on its hind limbs. The stretch-attend posture is a good indicator of exploratoryanxiety conflict situations, and this was assessed by the frequency with which the animal demonstrated forward elongation of the head and shoulders followed by retraction to the original position.

\section{Tissue processing for histological and histochemical analyses}

A day (24 hours) after the last treatment, rats for histological and histochemical studies (3 from each group) were euthanised by intramuscular injection of $50 \mathrm{mg} / \mathrm{kg}$ of ketamine and subjected to transcardial perfusion during which a flush of $50 \mathrm{ml}$ of normal saline was followed by $500 \mathrm{ml}$ of $4 \%$ paraformaldehyde (PFA). The brain tissues were thereafter excised and post-fixed in 4\% PFA for 48 hours and then processed for qualitative studies. PFA-fixed tissue sections were stained using Haematoxylin and Eosin (H\&E) and Cresyl Fast Violet staining techniques. Rats processed for enzymatic studies (5 from each group) were sacrificed by cervical dislocation (to eliminate the interference of anesthetic agent with biochemical redox); the brains were then excised, rinsed in $0.25 \mathrm{M}$ sucrose three times for 5 minutes each and stored in $30 \%$ sucrose at $4^{\circ} \mathrm{C}$.

\section{Biochemical assays for enzymatic analyses}

Enzymatic assay for malondialdehyde (MDA), superoxide dismutase (SOD), catalase (CAT), nitric oxide (NO), glutathione peroxidase (GPx), glucose-6-phosphate dehydrogenase (G6PDH), lactate dehydrogenase (LDH), Glucokinase, acetylcholinesterase (AChE), total protein (TP) and transferrin receptor protein (TRP) were quantified in brain tissues of rats using spectrophotometric techniques at the Central Research Laboratory, University Road, Ilorin, Kwara State, Nigeria. All assay kits of the aforementioned parameters were products of Abcam, USA, except indicated otherwise. Equal weighing brain tissues were homogenised in $0.25 \mathrm{M}$ sucrose at $40^{\circ} \mathrm{C}$. Tissue homogenate was centrifuged for 15 minutes at 5,000 rpm, after which the supernatant containing tissue lysate was aspirated into fresh tubes. The activities of proteins were assayed according to the manufacturer's instruction in the assay kit pack.

\section{Photomicrography and statistical analysis}

Photomicrographs of the cerebellum were obtained using an Amscope microscope camera attached to a light microscope. Quantitative analysis of the photomicrograph was done using Image J software (National Institutes of Health, Bethesda, MD, USA). The data obtained from the neurobehavioral and enzymatic assay were subjected to statistical analysis using Graph-pad Prism (version 6; Graph Pad, San Diego, CA, USA). The values were plotted in ANOVA with Tukey's multiple comparison tests. Data obtained were presented as mean \pm standard error of mean (SEM) with the level of significance set at a $P$-value less than 0.05 . The results obtained were represented in bar charts with error bars to show the mean and SEM, respectively.

\section{Results}

\section{ESO reversed $\mathrm{AlCl}_{3}$-induced dysregulation of general motor functions and exploratory drive in rats}

The most basic and common outcome of interest in the open-field test (OFT) is movement; however, this can be influenced by motor output, exploratory drive, anxiety levels, sickness, relative time in the circadian cycle, among many other variables [21]. In Fig. 1, a similar trend can be seen in the result between the PBS and ESO groups. Rats from these groups had a significantly higher frequency in the number of lines crossed (exploration and locomotion) (Fig. 1A) and in rearing behavior (Fig. 1B) respectively than rats from the 
A

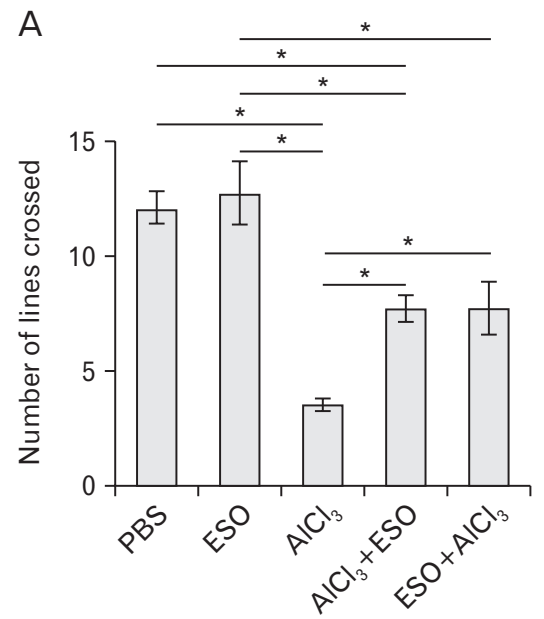

D

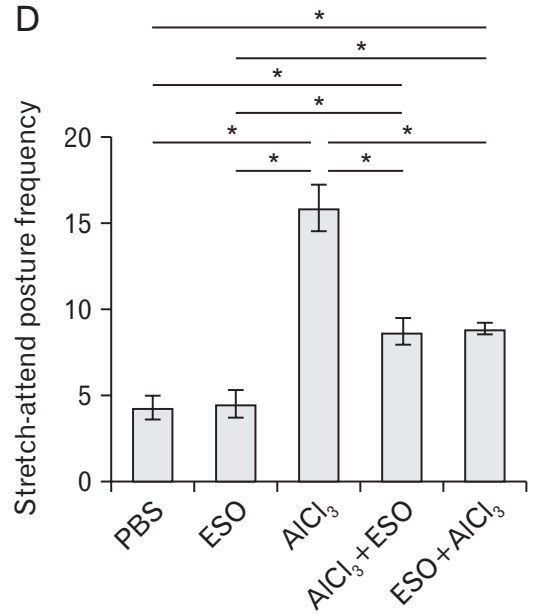

B

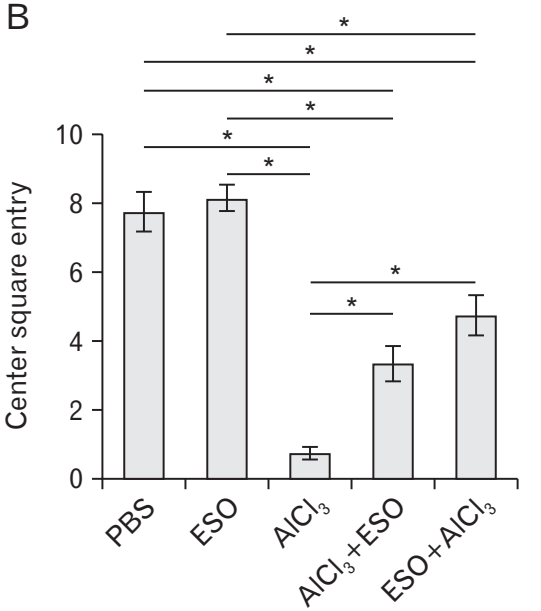

$E$

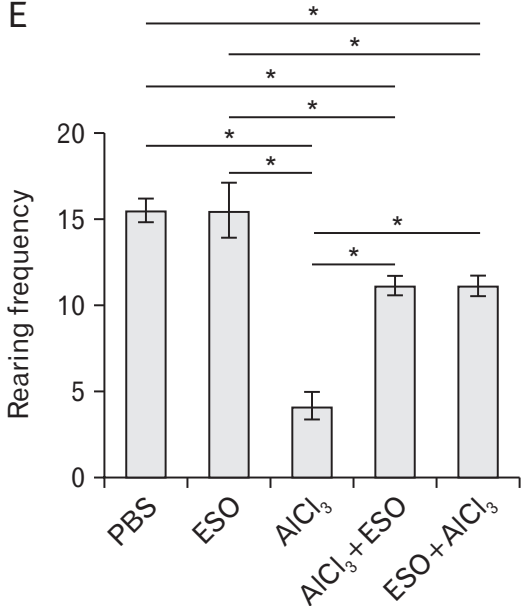

C
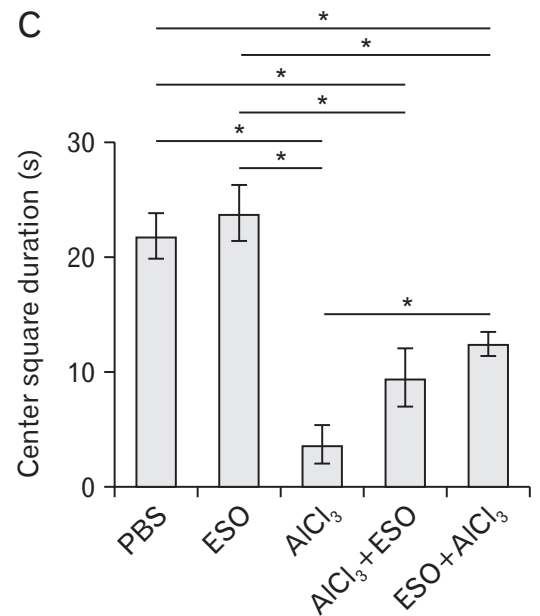

Fig. 1. (A-E) Effects of ESO administration on exploratory drive and locomotor activities in treated animals. (A) Analysis of the number of lines crossed by rats in the open field test showing no significant difference between the numbers of lines crossed by PBS and ESO controls. $\mathrm{AlCl}_{3}$ treated rats crossed the least number of lines relative to other groups. $\mathrm{AlCl}_{3}+\mathrm{ESO}$ and $\mathrm{ESO}+\mathrm{AlCl}_{3}$ groups significantly crossed more lines than the $\mathrm{AlCl}_{3}$ treated rats (both at $P<0.05$ ). (B) Analysis of central square entry frequency in the open field test showed that rats treated $\mathrm{AlCl}_{3}$ made the least entry to the central square of the open field compared to all of PBS $(P<0.05)$, ESO $(P<0.05), \mathrm{AlCl}_{3}+\mathrm{ESO}(P<0.05)$, and $\mathrm{ESO}+\mathrm{AlCl}_{3}$ $(P<0.05)$ groups. $(\mathrm{C})$ Analysis of central square duration revealed that the administration of $\mathrm{AlCl}_{3}$ reduced the time rats spent in the central square when compared to the PBS $(P<0.05)$ and ESO $(P<0.05)$ controls. However, ESO significantly improved the central square duration of rats in the $\mathrm{ESO}+\mathrm{AlCl}_{3}$ treated rats $(P<0.05)$ compared to the $\mathrm{AlCl}_{3}$ group. (D) Rearing frequency analysis of experimental animals showing significant reductions in both groups treated $\mathrm{AlCl}_{3}$ compared to other groups. ESO intervention improved rearing frequencies in rats in the $\mathrm{AlCl}_{3}+\mathrm{ESO}$ and $\mathrm{ESO}+\mathrm{AlCl}_{3}$ groups (at $P<0.05$, respectively) compared to the $\mathrm{AlCl}_{3}$ group. (E) Analysis of the stretch-attend posture frequency shows significant elevation in rats treated $\mathrm{AlCl}_{3}$ treated compared to $\mathrm{PBS}$ and $\mathrm{ESO}$ control (both at $P<0.05$ ). However, the $\mathrm{AlCl}_{3}+\mathrm{ESO}$ and $\mathrm{ESO}+\mathrm{AlCl}_{3}$ treated rats showed improved stretch-attend-posture, which is significantly different (both at $P<0.05$ ) from the $\mathrm{AlCl}_{3}$ group. The results are presented as mean \pm standard error of mean. $\mathrm{AlCl}_{3}$, aluminum chloride; $\mathrm{ESO}$, essential oil; PBS, phosphate-buffered saline. ${ }^{*} P<0.05$.

other two groups. High frequency of lines crossed and rearing is an indication of increased locomotion and exploration with a complimentarily lower level of anxiety. In addition, rats that received ESO following pretreatment with $\mathrm{AlCl}_{3}$ showed significant improvement in exploratory activities and rearing behavior $(P<0.05)$ than rats that received $\mathrm{AlCl}_{3}$ without ESO therapy. Subsequent analysis of the frequency of central square entry and duration of time spent in the central square (Fig. 1C, D) revealed identical results between the PBS and ESO groups, indicating high exploratory habit, emotional stability and lack of anxiety in rats so treated. However, rats in the $\mathrm{AlCl}_{3}$ group exhibited a lack of verve in the open field as they merely made entry to the central square or tarried within the square (indicative of anxiety and 
A

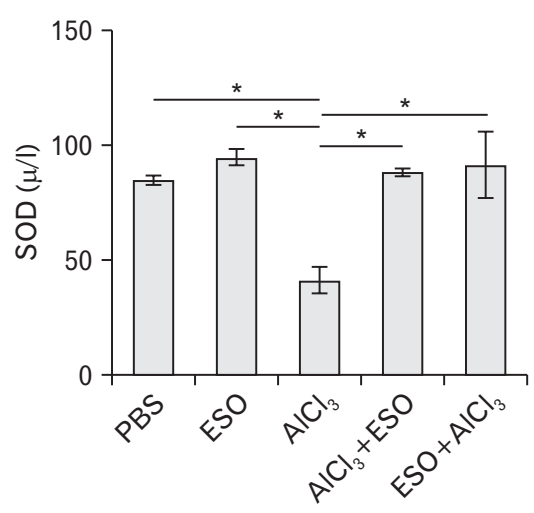

D

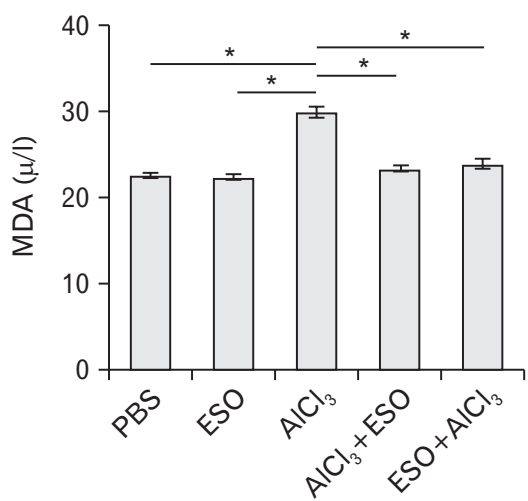

B

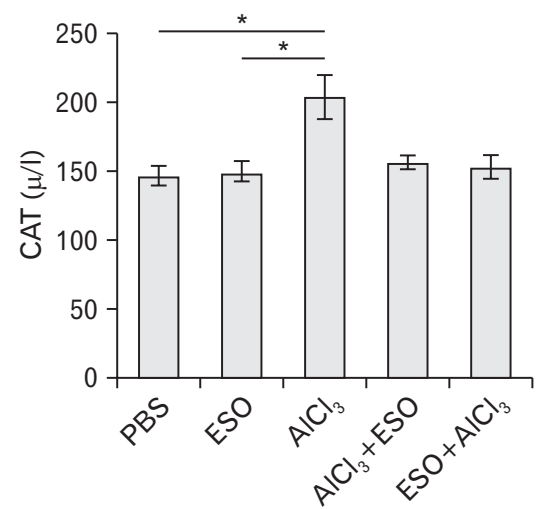

E

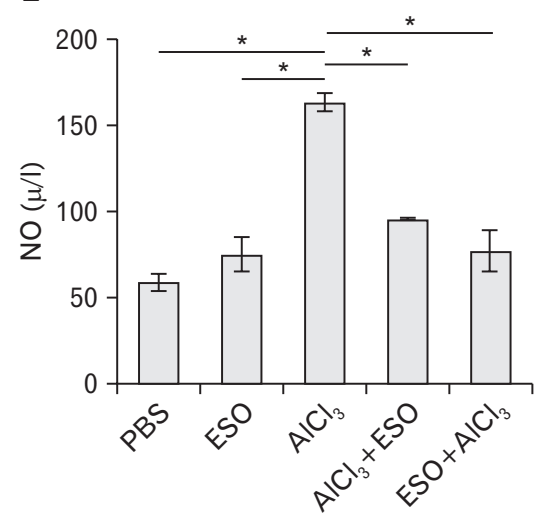

C

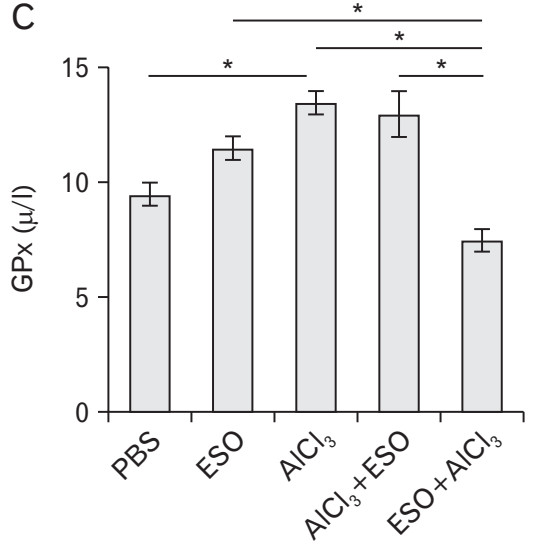

Fig. 2. Cerebellar oxidative redox state characterized by (A) SOD, (B) CAT, (C) GPx, (D) MDA, and (E) NO activities respectively. (A) Cerebellar SOD activity was significantly depleted by $\mathrm{AlCl}_{3}$ treatment relative to both PBS $(P<0.05)$ and ESO groups $(P<0.05)$ but was normalized by $\mathrm{ESO}$ treatment in $\mathrm{ESO}+\mathrm{AlCl}_{3}(P<0.05)$ and $\mathrm{AlCl}_{3}(P<0.05)$ groups. (B) CAT level was increased by $\mathrm{AlCl}_{3}$ relative to both PBS and $\mathrm{ESO}$ controls $(P<0.05)$ but regularized by ESO treatment in both $\mathrm{AlCl}_{3}+\mathrm{ESO}$ and $\mathrm{ESO}+\mathrm{AlCl}_{3}$ groups at $P<0.05$. (C) $\mathrm{AlCl}_{3}$ treatment induced an increase in GPx level relative to the PBS and ESO groups at $P<0.05$ for both. ESO treatment restored the level of GPx in $\mathrm{AlCl}_{3}+\mathrm{ESO}$ $(P<0.05)$ to normal levels but not $\mathrm{ESO}+\mathrm{AlCl}_{3}$ group. (D) $\mathrm{AlCl}_{3}$ treatment triggered a significant increase in the level of MDA when compared to the PBS $(P<0.05)$ and ESO $(P<0.05)$ controls. This was normalized in the $\mathrm{AlCl}_{3}+\mathrm{ESO}(P<0.05)$ and $\mathrm{ESO}+\mathrm{AlCl}_{3}(P<0.05)$ groups. $(\mathrm{C})$ $\mathrm{AlCl}_{3}$ treatment elicited a striking increase in NO activities relative to the PBS and ESO groups (both at $P<0.05$ ) and was regularized by ESO in both $\mathrm{AlCl}_{3}+\mathrm{ESO}$ and $\mathrm{ESO}+\mathrm{AlCl}_{3}$ groups (at $P<0.05$ each). $\mathrm{AlCl}_{3}$, aluminum chloride; CAT, catalase; $\mathrm{ESO}$, essential oil; GPx, glutathione peroxidase; MDA, malondialdehyde; NO, nitric oxide; PBS, phosphate-buffered saline; SOD, superoxide dismutase. ${ }^{*} P<0.05$.

poor motor functions), with entry frequency and duration of rats in the central square in the group significantly lower than in both PBS and ESO groups $(P<0.05)$. Similarly, both central square entry and duration of time spent in central square of rats in the $\mathrm{AlCl}_{3}+\mathrm{ESO}$ group were lower than seen in the PBS and ESO groups. However, the frequency of central square entry and duration in the group is significantly higher than in the $\mathrm{AlCl}_{3}$ group $(P<0.05)$. This result indicates that ESO improved exploratory behavior and lowered anxiety levels associated with $\mathrm{AlCl}_{3}$ neurotoxicity respectively. Additionally, observation of stretch-attend postures (SAP) in rats across the experimental groups revealed the significantly higher frequency of SAP in the $\mathrm{AlCl}_{3}$ group when compared with ESO and PBS groups (both at $P<0.005$ ) as shown in Fig. $1 \mathrm{E}$.

\section{Effects of ESO on $\mathrm{AlCl}_{3}$-induced perturbation of cerebellar redox}

Aluminum is known to cause neurotoxicity by exacerbating the production of reactive oxygen and nitrogen species. In this study, the $\mathrm{AlCl}_{3}$ group presented with significantly depleted level of SOD when compared to the control group $(P<0.05)$. Interestingly, the aluminum group presented with a raised level of CAT and GPx. This finding suggests that $\mathrm{AlCl}_{3}$ mediated a cascade of chemical event that rsulted in the elevated levels of superoxides, which led to a depletion 
in the level of SOD. To further examine the beneficial potentials of $\mathrm{ESO}$ in reversing $\mathrm{AlCl}_{3}$-induced oxidative stress, we measured cerebellar lysate levels of CAT, GPx, and SOD in the ESO pre and post-teated groups (Fig. 2). The results revealed that ESO significantly improved SOD activities in the $\mathrm{AlCl}_{3}+\mathrm{ESO}(P<0.05)$ and $\mathrm{ESO}+\mathrm{AlCl}_{3}(P<0.05)$ when compared to the $\mathrm{AlCl}_{3}$ group.

Furthermore, our findings revealed that $\mathrm{AlCl}_{3}$-treatment induced lipid peroxidation and nitrosative stress in the cerebellum of rats when compared to animals administered with PBS alone, as evidenced by elevated MDA $(P<0.05)$ and NO $(P<0.05)$ levels in cerebellar lysates. Significant decreases in levels of MDA and NO were recorded in groups pre- and post- exposed to $\mathrm{AlCl}_{3}$ subsequent to ESO administration, both at $P<0.05$ for MDA and NO (Fig. 2).

\section{Cerebellar G6PDH, LDH, and glucokinase expressions following $\mathrm{AlCl}_{3}$ and ESO treatments in rats}

Tissue levels of G6PDH were significantly reduced by $\mathrm{AlCl}_{3}$ treatment in the cerebellum $(P<0.005)$ relative to the PBS and ESO controls. Both pre- and post-administrations of ESOs were able to effectively counteract the neurotoxicity of $\mathrm{AlCl}_{3}$ by regulating cerebellar $\mathrm{G} 6 \mathrm{PDH}$ to baseline levels (Fig. 3A). To further ascertain the protective effect of ESO, we measured LDH expression within cerebellar lysates under different conditions. $\mathrm{AlCl}_{3}$ oral infusion significantly
A

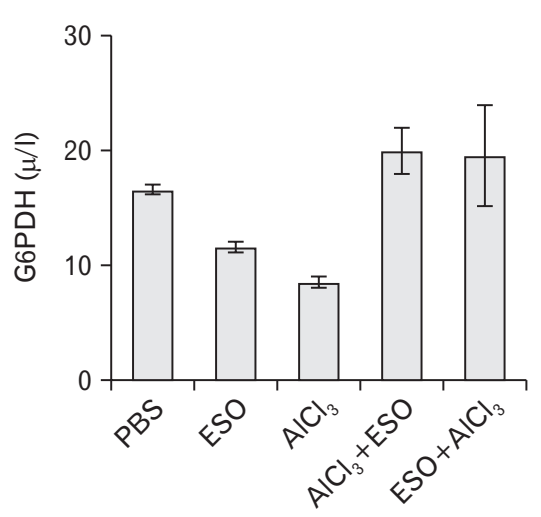

D

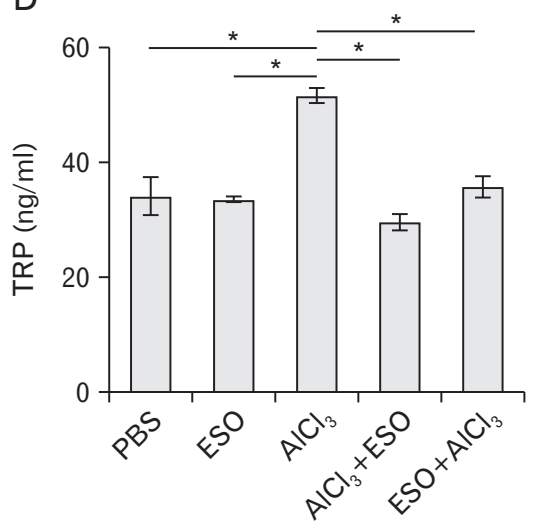

B

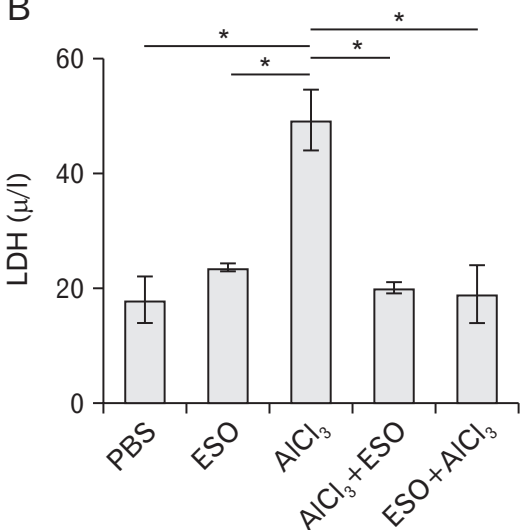

$\mathrm{E}$

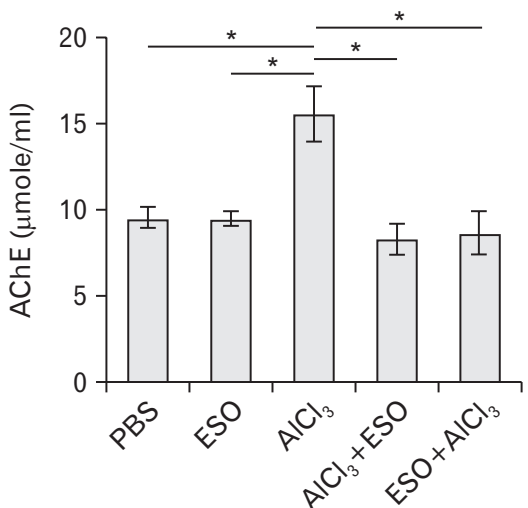

C

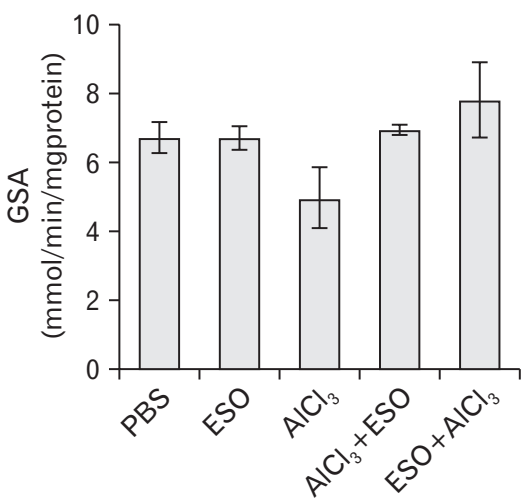

Fig. 3. Cerebellar G6PDH (A), LDH (B), GSA (C), TRP (D), and AChE (E) activities under treatment conditions. (A) $\mathrm{AlCl}_{3}$ treatment caused depletion in G6PDH expression relative to the PBS and ESO groups ( $P>0.05$ in both). Pre- and post-treatment with ESO normalized G6PDH levels in the $\mathrm{AlCl}_{3}+\mathrm{ESO}(P<0.05)$ and $\mathrm{ESO}+\mathrm{AlCl}_{3}(P<0.05)$ groups. (B) Expectantly, $\mathrm{AlCl}_{3}$ treatment induced a significant decrease in the level of LDH compared to the PBS, and ESO controls both at $P<0.05$. ESO treatment prevented LDH dysregulation in $\mathrm{AlCl}_{3}+\mathrm{ESO}(P<0.05)$ and $\mathrm{ESO}+\mathrm{AlCl}_{3}(P<0.05)$ treated rats. (C) Cerebral GSA activity was significantly depleted in $\mathrm{AlCl}_{3}$ treated rats when compared to PBS $(P<0.05)$, $\mathrm{ESO}(P<0.05), \mathrm{AlCl}_{3}+\mathrm{ESO}(P<0.05)$ and $\mathrm{ESO}+\mathrm{AlCl}_{3}(P<0.05)$ treated rats. (D) The expressed level of TRP in $\mathrm{AlCl}_{3}$ treated rats was significantly higher than in PBS, and ESO controls at $P<0.05$ each, as well as in $\mathrm{AlCl}_{3}+\mathrm{ESO}$ and $\mathrm{ESO}+\mathrm{AlCl}_{3}$ treated rats $($ both at $P<0.05)$. When compared to all other treated groups, $\mathrm{AlCl}_{3}$ treated rats presented with the highest level of $\mathrm{AChE}$, with significant modulation seen following ESO intervention in the $\mathrm{AlCl}_{3}+\mathrm{ESO}$ and $\mathrm{ESO}+\mathrm{AlCl}_{3}$ both at $P<0.05$. $\mathrm{AChE}$, acetylcholinesterase; $\mathrm{AlCl}$, aluminum chloride; $\mathrm{ESO}$, essential oil; GSA, glucokinase specific activity; G6PDH, glucose-6-phosphate dehydrogenase; LDH, lactate dehydrogenase; PBS, phosphatebuffered saline; TRP, transferrin receptor protein. ${ }^{*} P<0.05$. 
increased LDH activity within the cerebellum, which is an index of neurotoxicity (Fig. 3B). We found ESO to be significantly effective in normalizing LDH expression to baseline levels in both $\mathrm{AlCl}_{3}+\mathrm{ESO}(P<0.05)$ and in the $\mathrm{ESO}+\mathrm{AlCl}_{3}$
$(P<0.05)$ groups. Further analysis showed that $\mathrm{AlCl}_{3}$ treatment depleted the level of Glucokinase in cerebellar lysates, although changes were insignificant statistically (Fig. 4). ESO treatment was, however, able to restore the level of Glucoki-

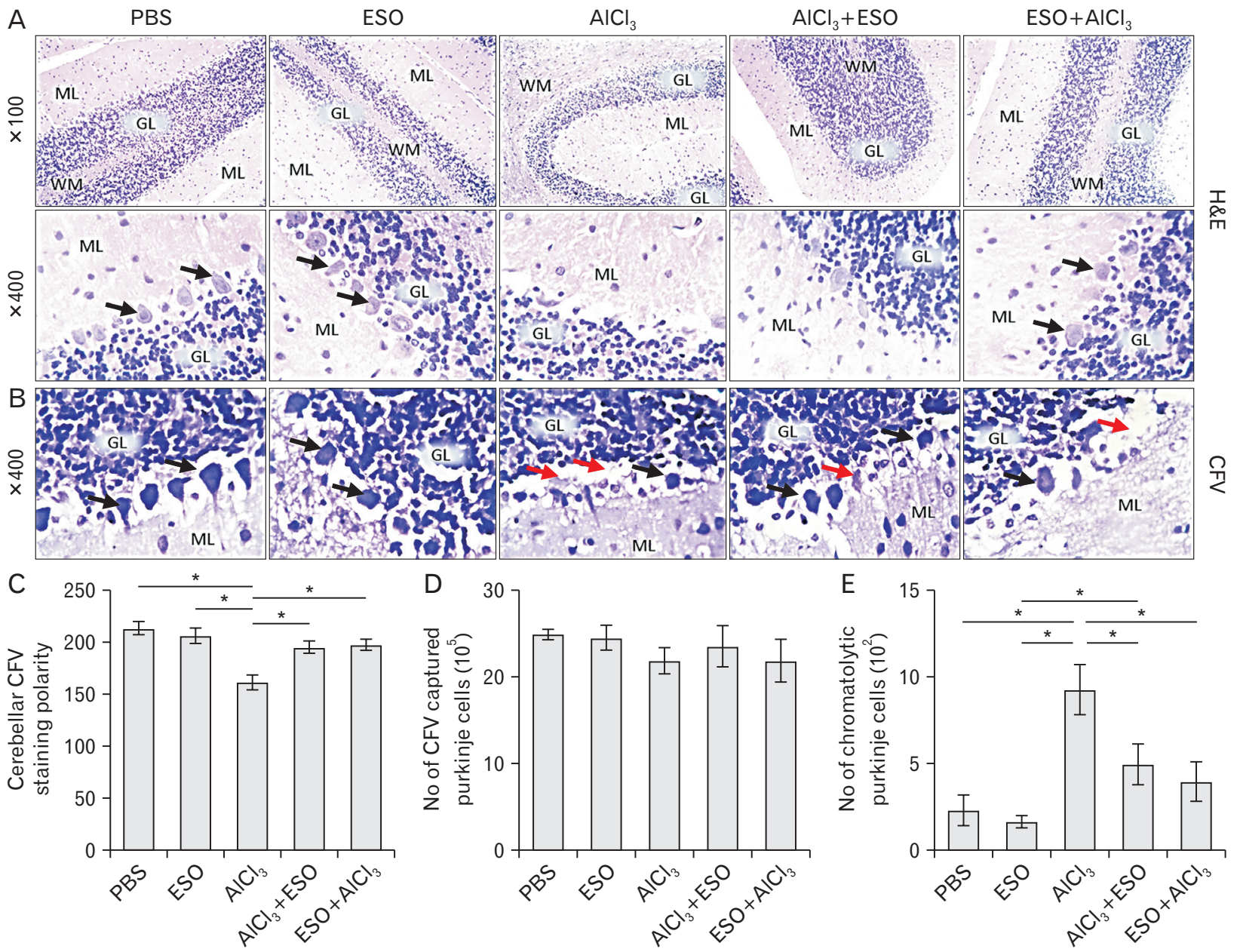

Fig. 4. (A-E) Representative photomicrographs showing panoramic views of cerebellar cortex general morphological presentations and Nissl profile in Wistar rats across the various study groups. (A) is H\&E ( $\times 100, \times 400)$; The ML, Purkinje cells (black arrowhead) layer, GL, and the medullary layer of WM are demonstrated across study groups. PBS and ESO treatments did not alter the morphological presentation of cerebellar layers from this study. Both groups show a fine array of cells that are distinctly arranged from the molecular to the granular layer. Also, cellular density within the groups appears normal across all cortical layers. $\mathrm{AlCl}_{3}$ treatment caused degenerative changes characterized by fragmented GL in cerebellar cortex and poorly stained Purkinje cells. Comparatively, increased cellular density was observed in this group. Groups administered ESO before and after $\mathrm{AlCl}_{3}$-induced toxicity $\left(\mathrm{AlCl}_{3}+\mathrm{ESO}\right.$ and $\left.\mathrm{ESO}+\mathrm{AlCl}_{3}\right)$ shows mild degenerative changes in line of morphologic appearance of cerebellar sections of $\mathrm{AlCl}_{3}$-treated rats; cerebellar layers in both groups looks better structured and layers are well-delineated. (B) shows Nissl profile as demonstrated by CFV stain $(\times 400)$. Normal morphological presentations of the cerebellar cortex are observable in PBS and ESO treated groups that are characterized by well-defined staining profiles. Cellular morphology in these groups is characterized by Purkinje cells with conspicuous cell bodies and dendrites that are projecting deep into the MLs (black arrowhead). Also, the granule layer in these groups consists of small granule neurons which are well outlined in contrast to the loosely arranged and cryptic cells in the granule layers of groups $\mathrm{AlCl}_{3}$ treated groups. Degenerating Purkinje cells with chromatolytic cell bodies (red arrowhead) and short dendritic processes can be seen around the indistinctly demarcated cerebellar layers of groups that receive aluminum treatment. The transitional regions between the cerebellar layers of $\mathrm{AlCl}_{3}$ treated with ESO are also generally better delineated. Although a few perineural spaces are present around Purkinje neurons of this groups, the general cytoarchitecture is much similar to that of the PBS and ESO groups. (C-E) show quantitative analysis of the CFV stain. $\mathrm{AlCl}_{3}$, aluminum chloride; CFV, Cresyl Fast Violet; ESO, essential oil; GL, granule cell layer; ML, molecular layer; PBS, phosphate-buffered saline; WM, white matter. ${ }^{*} P<0.05$. 
nase in the cerebellum back to basal levels, i.e. as compared with the control (PBS and ESO).

\section{ESO Modulates AChE and TRP activities following $\mathrm{AlCl}_{3}$-induced neurotoxic cascades}

The loss of cholinergic neurotransmission in the cerebral cortex and other areas contribute significantly to deterioration of cognitive function seen in patients with $\mathrm{AD}$. AChE interferes with choline uptake/release at presynaptic terminals, resulting in an alteration of neurotransmission evidenced by substantial neocortical deficits in the enzyme responsible for the synthesis of acetylcholine (ACh), choline acetyltransferase (ChAT). We observed elevated levels of $\mathrm{AChE}$ in $\mathrm{AlCl}_{3}$-treated groups (Fig. $4 \mathrm{~A}$ ) relative to both $\mathrm{PBS}$ and ESO controls. Similar to earlier results of this study, we found that pre- and post- treatment of rats with ESO following $\mathrm{AlCl}_{3}$-induced neurotoxicity significantly downregulated AChE levels to basal levels (both at $P<0.01$ ). In rats that received $\mathrm{AlCl}_{3}$, we further observed a marked increase in the level of TRP (Fig. 5C) when compared to baseline levels in the controls (PBS and ESO). The therapeutic potentials of ESO were further buttressed in this study by its normoregulating roles in expressed TRP in cerebellar homogenates, wherein animals post-treated with ESO following $\mathrm{AlCl}_{3}$ infusion $\left(\mathrm{AlCl}_{3}+\mathrm{ESO}\right)$ showed significantly decreased TRP expression in comparison to the $\mathrm{AlCl}_{3}(P<0.05$ and $P<0.01$ respectively).

\section{Cerebellar histology and nissl profile following $\mathrm{AlCl}_{3^{-}}$ induce toxicity and $d$ ESO therapy}

The cerebellum possesses distinctive histological appearance that can become invariably altered secondary to inflammatory reactions and oxidative stress dysregulation. We comparatively examined thin sections of cerebellar tissues using routine H\&E and Nissl staining methods. Panoramic representation of cerebellar histological profile of the control groups (PBS and ESO) was regular, with consistent layering and succinct transitions seen between molecular, granular and Purkinje neuron layers (Fig. 4A). Similarly, a close examination of the Purkinje cells using a higher magnification, showed that neither of PBS nor ESO treatment altered the structural integrity of these all-important cerebellar neurons. Additionally, apical and basal dendrites of neurons were well expressed and interconnected within the neuropil of both control groups (Fig. 5A). As anticipated, the cerebellar milieu of $\mathrm{AlCl}_{3}$-treated rats were characterised by the extravasation of the granule cells into the Purkinje cell layer, fragmentation of neuropil, distortion of layering, and cytoplasmic degeneration of neurons. Furthermore, there was a marked reduction in the deposition of Nissl proteins in cerebellar sections of these groups when compared to rats that received PBS and ESO, which is indicative of chromatolytic changes via alteration in protein synthesis (Fig. 4B). Quantitative analysis of the Nissl stain revealed that the aluminium group had a significantly reduced staining intensity when compared to the control group $(P<0.05)$ (Fig. 4C). Also, relative to the control group, the $\mathrm{AlCl}_{3}$ group presented with more chromatolytic Purkinje neurons (Fig. 4E). Interestingly, both treatments with ESO before and after $\mathrm{AlCl}_{3}$ induced degenerative changes on the cerebellum improved general histomorphology and Nissl profiles, as seen in both $\mathrm{AlCl}_{3}+\mathrm{ESO}$ and $\mathrm{ESO}+\mathrm{AlCl}_{3}$ groups (Fig. 4B). The $\mathrm{AlCl}_{3}+\mathrm{ESO}$ and $\mathrm{ESO}+\mathrm{AlCl}_{3}$ groups also presented with an increase in staining intensity and reduced Purkinje chromatolysis when compared to the $\mathrm{AlCl}_{3}$ group. The finding suggests that ESO can restore/maintain histomorphological integrity to the cerebellum following $\mathrm{AlCl}_{3}$ induced neurotoxicity.

\section{Discussion}

This study showed the possible neuroprotective/neuromodulatory potentials of ESO before and after $\mathrm{AlCl}_{3}$ exposure on rat's cerebellar morphology and functions through behavioral, biochemical, and histoarchitectural evaluations. The use of neurobehavioral studies in risk assessment is premised on the fact that behavior/emotions can be regarded as the net output of memory, motor, locomotor and cognitive functions occurring in the nervous system and can serve as potentially sensitive endpoints of chemically induced-neurotoxicity [22]. $\mathrm{AlCl}_{3}$ is a neurotoxicant that permeates and functionally alters the blood-brain barrier and potentially produces changes in the ionic, cholinergic, dopaminergic, and noradrenergic neurotransmission in the central nervous system. These alterations are known to be associated with impairment of visuoperception, attention, cognitive, memory, and learning ability $[23,24]$. Data obtained from the OFT showed a decrease in exploratory activities of rats treated with $\mathrm{AlCl}_{3}$, as seen by a significant decline in the number of lines crossed, rearing frequency, center square entry, center square duration, and elevated SAP when compared with other groups. Reports of decreased exploratory and locomotor activities have been reported following $\mathrm{AlCl}_{3}$ 
treatment. Our lab and others from literature have reported decreased crossing scores and altered secondary parameters such as rearing, grooming, and sniffing in the OFT following $\mathrm{AlCl}_{3}$ treatment $[25,26]$. Decreased locomotor activity assessed by actophotometer following treatment with $\mathrm{AlCl}_{3}$ was reported, and it was deduced from this study that this effect might be due to possible depressant effects of $\mathrm{AlCl}_{3}$ on the CNS [23]. A significant reduction has been documented in the exploratory habits of rats treated with sodium azideanother mitochondrial toxin with neurotoxicant properties [27]. Suggestively, the altered behavioral pattern seen in the $\mathrm{AlCl}_{3}$ group in this study might be due to its ability to impair glucose utilisation and the release of cytochrome $\mathrm{C}$, which inhibits the production of ATP generation. This hypothesis is supported by findings of glucose bioenergetics dysfunction seemingly caused by $\mathrm{AlCl}_{3}$ treatment in this study. ESO treatment before and after $\mathrm{AlCl}_{3}$ toxicity significantly improved exploratory and locomotor outcomes in the OFT. It is noteworthy that treatment with ESO before $\mathrm{AlCl}_{3}$ treatment was more effective than after, indicating that ESO is more effective in a preventive/pro-adaptive/protective role. Improved glucose biosynthesis parameters in the cerebellum of rats treated with ESO following $\mathrm{AlCl}_{3}$ toxicity may underlie its therapeutic and protective efficacy against motor-related behavioral alterations in this study.

The mechanism of cytotoxic and/or neurotoxic substances are thought to be mainly due to the oxidative stress involved in the production of reactive oxygen species (ROS), including superoxide anion, hydrogen peroxide, superoxide radical and hydroxyl radical. Moreover, the degree of oxidative damage is depended on the balance between the oxidative stress and the efficiency of the endogenous antioxidant system that is found in the majority of cells [5]. Generally, brain tissues are highly susceptible to attacks from free radicals due to highly unsaturated lipid contents [28]. Studies have shown that exposure to $\mathrm{Al}$ results in the impairment of mitochondrial functions in vivo and in vitro, as well as destroying the antioxidant defense system by decreasing the antioxidant enzyme status [29]. We, therefore, measured lipid peroxidation by assessing MDA levels, nitrosative stress by quantifying $\mathrm{NO}$ and determining status of endogenous antioxidant enzymes, including SOD, CAT, and GPx following $\mathrm{AlCl}_{3}$-induced cerebellar toxicity while studying the possible neuroprotective mechanisms of ESO. Since lipid oxidation products are one of the primary outcome associated with oxidative stress, the significantly increased cerebellar level of MDA in the group treated with $\mathrm{AlCl}_{3}$ in this study reflect induction of lipid production by the neurotoxicant. This result corresponds with other studies in which $\mathrm{Al}$ treatment increased iron-dependent lipid peroxidation in rat brain [30], and especially in cerebrum and cerebellum following intraperitoneal treatment of $\mathrm{Al}$ [26]. In this study, rats treated with ESO before and after $\mathrm{AlCl}_{3}$ intoxication show marked reduction of MDA level in the cerebellar homogenate. Cymbopogon citratus has been reported to possess flavonoids, such as luteolin, apigenin, glycosides and tannins and these actively contributed to its antioxidant and anti-inflammatory properties [18], and this may underlie its ability to reduce lipid peroxidation in this study.

Overproduction of $\mathrm{NO}$ makes it noxious and can undergo oxidative-reductive reactions if a cell is in a pro-oxidant state to form toxic compounds (reactive nitrogen species', or RNS), which cause cellular damage [31,32]. Recently, the term 'nitrosative stress' has been used to indicate the cellular damage that is elicited by excess NO and RNS (peroxynitrite and nitrogen [III] oxide), and have been implicated in the pathogenesis of neurodegenerative disorders. Nitrotyrosine formation, a marker of nitrosative stress, has been reported both in AD and Parkinson's patients [33]. As a response to $\mathrm{AlCl}_{3}$-induced neurotoxicity in this study, we observed significant upregulation of NO in the cerebellar cortex of rats, which might be due to the ability of Al to selectively favor the production of NO from l-arginine via the activation of $\mathrm{NO}$ synthase. Interestingly, we found that rats treated with ESO before and after $\mathrm{AlCl}_{3}$ intoxication shows normal $\mathrm{NO}$ level in cerebellar lysates, which correlates with improved behavioural outcome.

This observation was further buttressed by results from SOD, CAT, and GPx analyses. Recent evidence suggests the formation of superoxide anion and expression/activity of its endogenous scavenger-SOD as a common player in the pathogenic cascades of many neurodegenerative pathologies like AD [5]. Similarly, GPx an enzyme that reduces lipid hydroperoxides to their corresponding alcohols and reduces free hydrogen peroxide to water, and CAT, which protects SOD by converting $\mathrm{H}_{2} \mathrm{O}_{2}$ to water and oxygen, have both been implicated in AD etiology. Our findings showed that treatment with Al evidently increases oxidative stress and brain injury as there was significant repression in the levels of SOD, GPx, and CAT in the cerebellar homogenate relative to control. These results corroborated previous findings that Al treatment results to downregulation of antioxidant 
enzymes, causing an imbalance between pro-oxidant and antioxidant potentials $[5,26]$. On the contrary, the administration of ESO before and after Al treatment showed a reversal of the deleterious effects on oxidative redox within the cerebellum of rats. Flavonoids are known to exert their protective effect by directly scavenging ROS, by activating antioxidant enzymes or through metal chelating activity. These may account for the relative restoration of homeostasis shown by ESO in this study.

Disturbances in central or peripheral glucose bioenergetics, which can be affected by almost every level of cellular or biochemical metabolic cascades have been implicated in various neurodegenerative pathologies [5]. LDH and G6PDH are enzymes in the energy-producing glycolytic pathway, which may be affected by oxidative modification and decreased activity may contribute to the alteration in glucose metabolism noted in $\mathrm{AD}$ [34]. The consequence of loss of neuronal membrane integrity due to oxidative injury is the release of $\mathrm{LDH}$ in a large quantity. Its aberrant production in cells is a useful tool for determining cytotoxicity and in measuring early cellular damage or impairment [35]. The cerebellar cortex of rats treated $\mathrm{AlCl}_{3}$ showed significant upregulation of $\mathrm{LDH}$ activities in the cerebellar cortex when compared to the control group. Reports of LDH alteration in the cerebellar cortex following treatment of rat with $\mathrm{Al}$ have been documented, more than any metabolically active organ, LDH gene expression ratio in the brain is the most altered in response to impaired oxidative phosphorylation [36]. Alteration of cerebellar LDH levels in this study may also be linked to oxidative stress caused by excess production of ROS and RNS induced by $\mathrm{Al}$ treatment. Rats in this group also experienced a decline in energy and ribose production through pentose phosphate pathways, as evidenced by decreased G6PDH in cerebellar homogenates. These findings indicate that one mechanism through which $\mathrm{Al}$ induces its toxicity is by altering energy production through inhibition of the activities of key enzymes responsible for glucose metabolism and that ESO has both protective and therapeutic effects against dysfunction of cerebellar bioenergetics. Transferrin is known primarily for its role in the transport and cellular uptake of iron but is also the major serum binding protein for aluminum [37]. One of the mechanisms through which $\mathrm{Al}$ exerts its neurotoxicity is its ability to hinder iron transport and absorption by displacing iron from binding to transferrin and therefore inhibit iron metabolism [38]. We investigated cerebellar levels of TRP following the oral infusion of $\mathrm{Al}$ and
ESO in Wistar rats. $\mathrm{AlCl}_{3}$ upregulated TRP in the cerebellar of rats, which may be due to the ability of $\mathrm{Al}$ to prevent the binding of iron to transferrin resulting into the accumulation of TRP and subsequently iron-induced oxidative stress. The potentials shown by ESO in normalising aberrant TRP expression is an underpinning of its therapeutic mechanism in this study. ESO's ability to prevent the accumulation of transferrin maybe by inhibiting $\mathrm{Al}$ from displacing iron in the iron-transferrin complex and subsequent iron-induced oxidative stress, which is crucial to inhibiting eventual neurotoxic cascades.

AChE plays a crucial role in learning, memory, and cognitive functions [23]. Cholinergic neurons are positive markers for the evolution of memory and related disorders affecting $\mathrm{ACh}$, and impaired cholinergic transmission is evident in various neurological diseases associated with cognitive impairment $[24,26]$. In addition to findings of the present study, cholinotoxic effects exerted by $\mathrm{AlCl}_{3}$ has been shown in previous studies to produce a functional change in cholinergic and noradrenergic neurotransmission. Such toxic changes to the neurotransmission system in the cerebellum may account for the loss of locomotor activities that were recorded in the open field, as we have found. Also, the ability of ESO to mitigate AChE upregulation in the cerebellum is an important aspect of its ability to restore and prevent locomotor irregularities. An interplay between increased tissue ROS and alteration of AChE synthesis has been described [23], spotlighting a possible bidirectional mechanism of action for ESO in the present study.

Histological observations showed that the cerebellar cortex in rats treated $\mathrm{AlCl}_{3}$ is characterised by fragmented granule cell layers with distorted neuropil, pyknotic Purkinje cells, and increased cellular density. Therefore, inhibition of the demonstrated cytotoxic impact of $\mathrm{AlCl}_{3}$ by ESO treatment is also a key component of its therapeutic potentials. Additionally, Nissl integrity in granule and Purkinje neurons within the cerebellum of $\mathrm{AlCl}_{3}$ treated rats show highly chromatolytic changes compared to controls, which indicates abnormal normal endoplasmic functioning and intercellular protein synthesis - corroborating results obtained from TP biochemical analysis.

Nissl granules are known to change under physiological and pathological conditions where they may dissolve and disappear in a condition known as chromatolysis, which can be triggered by axotomy, ischemia, and toxicity to the cell, as well as cell exhaustion or virus infections leading to disinte- 
gration [39]. If not checked, chromatolysis often resuly in cell death and ultimately neurodegeneration. Our present finding is in agreement with previous reports that highlighted the neurodegenerative impact of $\mathrm{Al}$ on the Nissl bodies in neurons $[40,41]$. Al-induced trauma to protein macromolecules and induced endoplasmic reticular (ER) stress in neurons may have resulted in chromatolysis and progressive decline in normal cerebellar histomorphology and function. The observed chromatolytic changes in the Purkinje neurons may explain the behavioral decline of the animals in the OFT. Therefore, the role of ESO to prevent and reverse chromatolysis in cerebellar neurons in this study may stem from its ability to prevent ER stress and inhibiting pathways that lead to aberrant protein production owing to its antioxidative and ROS scavenging properties.

In conclusion, summarily, our results strengthen the idea that $\mathrm{AlCl}_{3}$ initiates neuronal toxicity and degeneration in the cerebellum through the initiation of oxidative stress, as seen by the excessive production of free radicals and depleted endogenous antioxidant system, which resulted in neuronal damage. It is, however, intriguing that these degenerative changes were pharmacologically mitigated following ESO administration and should, therefore, be explored further towards the development of novel therapy for neurodegeneration.

\section{ORCID}

\author{
Gbadamosi Ismail Temitayo: \\ https://orcid.org/0000-0002-1932-7519 \\ Bamisi Olawande: https://orcid.org/0000-0001-7625-2456 \\ Yawson Olushola Emmanuel: \\ https://orcid.org/0000-0001-9482-7102 \\ Arogundade Tolulope Timothy: \\ https://orcid.org/0000-0002-0179-6181 \\ Ogunrinola Kehinde: \\ https://orcid.org/0000-0002-0488-4988 \\ Lewu Folashade Susan: \\ https://orcid.org/0000-0002-6305-6277 \\ Lambe Ezra: https://orcid.org/0000-0003-0819-6872 \\ Olajide Olayemi Joseph: \\ https://orcid.org/0000-0002-9259-2837
}

\section{Author Contributions}

Conceptualization: OOJ. Data acquisition: GIT, OOJ.
Data analysis or interpretation: GIT, ATT. Drafting of the manuscript: GIT, BO, YOE, ATT. Critical revision of the manuscript: OK, LFS, LE, OOJ. Approval of the final version of the manuscript: all authors.

\section{Conflicts of Interest}

No potential conflict of interest relevant to this article was reported.

\section{References}

1. Nelson PT, Alafuzoff I, Bigio EH, Bouras C, Braak H, Cairns NJ, Castellani RJ, Crain BJ, Davies P, Del Tredici K, Duyckaerts C, Frosch MP, Haroutunian V, Hof PR, Hulette CM, Hyman BT, Iwatsubo T, Jellinger KA, Jicha GA, Kövari E, Kukull WA, Leverenz JB, Love S, Mackenzie IR, Mann DM, Masliah E, McKee AC, Montine TJ, Morris JC, Schneider JA, Sonnen JA, Thal DR, Trojanowski JQ, Troncoso JC, Wisniewski T, Woltjer RL, Beach TG. Correlation of Alzheimer disease neuropathologic changes with cognitive status: a review of the literature. J Neuropathol Exp Neurol 2012;71:362-81.

2. Mota SI, Ferreira IL, Rego AC. Dysfunctional synapse in Alzheimer's disease - a focus on NMDA receptors. Neuropharmacology 2014;76 Pt A:16-26.

3. Hardy J, Selkoe DJ. The amyloid hypothesis of Alzheimer's disease: progress and problems on the road to therapeutics. Science 2002;297:353-6.

4. Wu T, Hallett M. The cerebellum in Parkinson's disease. Brain 2013;136(Pt 3):696-709.

5. Olajide OJ, Ugbosanmi AT, Enaibe BU, Ogunrinola KY, Lewu SF, Asogwa NT, Akapa T, Imam A, Ibrahim A, Gbadamosi IT, Yawson EO. Cerebellar molecular and cellular characterization in rat models of Alzheimer's disease: neuroprotective mechanisms of Garcinia biflavonoid complex. Ann Neurosci 2017;24:32-45.

6. Mathiyazahan DB, Thenmozhi AJ, Manivasagam T. Protective effect of black tea extract against aluminium chloride-induced Alzheimer's disease in rats: a behavioural, biochemical and molecular approach. J Funct Foods 2015;16:423-35.

7. Rodella LF, Ricci F, Borsani E, Stacchiotti A, Foglio E, Favero G, Rezzani R, Mariani C, Bianchi R. Aluminium exposure induces Alzheimer's disease-like histopathological alterations in mouse brain. Histol Histopathol 2008;23:433-9.

8. Kumar A, Prakash A, Dogra S. Neuroprotective effect of carvedilol against aluminium induced toxicity: possible behavioral and biochemical alterations in rats. Pharmacol Rep 2011;63:915-23.

9. Roskams AJ, Connor JR. Aluminum access to the brain: a role for transferrin and its receptor. Proc Natl Acad Sci U S A 1990;87:9024-7.

10. Crapper DR, Krishnan SS, Quittkat S. Aluminium, neurofibril- 
lary degeneration and Alzheimer's disease. Brain 1976;99:67-80.

11. McDonald B, Esiri MM, Morris J. Aluminium and Alzheimer's disease. Age Ageing 1993;22:392-3.

12. Adeneye AA, Agbaje EO. Hypoglycemic and hypolipidemic effects of fresh leaf aqueous extract of Cymbopogon citratus Stapf. in rats. J Ethnopharmacol 2007;112:440-4.

13. Tapia A, Cheel J, Theoduloz C, Rodríguez J, SchmedaHirschmann G, Gerth A, Wilken D, Jordan M, Jiménez-González E, Gomez-Kosky R, Mendoza EQ. Free radical scavengers from Cymbopogon citratus (DC.) stapf plants cultivated in bioreactors by the temporary immersion (TIS) principle. Z Naturforsch C J Biosci 2007;62:447-57.

14. Orrego R, Leiva E, Cheel J. Inhibitory effect of three C-glycosylflavonoids from Cymbopogon citratus (Lemongrass) on human low density lipoprotein oxidation. Molecules 2009;14:3906-13.

15. Campos J, Schmeda-Hirschmann G, Leiva E, Guzmán L, Orrego R, Fernández P, González M, Radojkovic C, Zuñiga FA, Lamperti L, Pastene E, Aguayo C. Lemon grass (Cymbopogon citratus (D.C) Stapf) polyphenols protect human umbilical vein endothelial cell (HUVECs) from oxidative damage induced by high glucose, hydrogen peroxide and oxidised low-density lipoprotein. Food Chem 2014;151:175-81.

16. Salim E, Kumolosasi E, Jantan I. Inhibitory effect of selected medicinal plants on the release of pro-inflammatory cytokines in lipopolysaccharide-stimulated human peripheral blood mononuclear cells. J Nat Med 2014;68:647-53.

17. Francisco V, Costa G, Figueirinha A, Marques C, Pereira P, Miguel Neves B, Celeste Lopes M, García-Rodríguez C, Teresa Cruz M, Teresa Batista M. Anti-inflammatory activity of Cymbopogon citratus leaves infusion via proteasome and nuclear factor- $\kappa \mathrm{B}$ pathway inhibition: contribution of chlorogenic acid. J Ethnopharmacol 2013;148:126-34.

18. Figueirinha A, Paranhos A, Pérez-Alonso JJ, Santos-Buelga C, Batista MT. Cymbopogon citratus leaves: characterization of flavonoids by HPLC-PDA-ESI/MS/MS and an approach to their potential as a source of bioactive polyphenols. Food Chem 2008;110:718-28.

19. Garcia R, Ferreira JP, Costa G, Santos T, Branco F, Caramona M, de Carvalho M, Dinis AM, Batista MT, Castel-Branco M, Figueiredo IV. Evaluation of anti-inflammatory and analgesic activities of cymbopogon citratus in vivo-polyphenols contribution. Res J Med Plant 2015;9:1-13.

20. Ganjewala D. Cymbopogon essential oils: chemical compositions and bioactivities. Int J Essent Oil Ther 2009;3:56-65.

21. Stanford SC. The open field test: reinventing the wheel. J Psychopharmacol 2007;21:134-5.

22. Nehru B, Bhalla P. Biochemical alterations in rat brain following aluminum exposure: protection with centrophenoxine. Toxicol Environ Chem 2007;89:577-85.

23. Amjad S, Umesalma S. Protective effect of Centella asiatica against aluminium-induced neurotoxicity in cerebral cortex, striatum, hypothalamus and hippocampus of rat brain histopathological, and biochemical approach. J Mol Biomark Diagn 2015;6:212.
24. Lakshmi BV, Sudhakar M, Prakash KS. Protective effect of selenium against aluminum chloride-induced Alzheimer's disease: behavioral and biochemical alterations in rats. Biol Trace Elem Res 2015;165:67-74.

25. Olajide OJ, Fatoye JO, Idowu OF, Ilekoya D, Gbadamosi IT, Gbadamosi MT, Asogwa NT. Reversal of behavioral decline and neuropathology by a complex vitamin supplement involves modulation of key neurochemical stressors. Environ Toxicol Pharmacol 2018;62:120-31.

26. Taïr K, Kharoubi O, Taïr OA, Hellal N, Benyettou I, Aoues A. Aluminium-induced acute neurotoxicity in rats: treatment with aqueous extract of Arthrophytum (Hammada scoparia). J Acute Disease 2016;5:470-82.

27. Olajide OJ, Enaibe BU, Bankole OO, Akinola OB, Laoye BJ, Ogundele OM. Kolaviron was protective against sodium azide $(\mathrm{NaN} 3)$ induced oxidative stress in the prefrontal cortex. Metab Brain Dis 2016;31:25-35.

28. Kumar V, Gill KD. Oxidative stress and mitochondrial dysfunction in aluminium neurotoxicity and its amelioration: a review. Neurotoxicology 2014;41:154-66.

29. Sayre LM, Perry G, Smith MA. Oxidative stress and neurotoxicity. Chem Res Toxicol 2008;21:172-88.

30. Yuan CY, Lee YJ, Hsu GS. Aluminum overload increases oxidative stress in four functional brain areas of neonatal rats. J Biomed Sci 2012;19:51.

31. Guix FX, Uribesalgo I, Coma M, Muñoz FJ. The physiology and pathophysiology of nitric oxide in the brain. Prog Neurobiol 2005;76:126-52.

32. Pacher P, Beckman JS, Liaudet L. Nitric oxide and peroxynitrite in health and disease. Physiol Rev 2007;87:315-424.

33. Calabrese V, Mancuso C, Calvani M, Rizzarelli E, Butterfield DA, Stella AM. Nitric oxide in the central nervous system: neuroprotection versus neurotoxicity. Nat Rev Neurosci 2007;8:766-75.

34. Chen Z, Zhong C. Decoding Alzheimer's disease from perturbed cerebral glucose metabolism: implications for diagnostic and therapeutic strategies. Prog Neurobiol 2013;108:21-43.

35. Zhang X, Yang F, Xu C, Liu W, Wen S, Xu Y. Cytotoxicity evaluation of three pairs of hexabromocyclododecane (HBCD) enantiomers on Hep G2 cell. Toxicol In Vitro 2008;22:1520-7.

36. Ross JM, Öberg J, Brené S, Coppotelli G, Terzioglu M, Pernold K, Goiny M, Sitnikov R, Kehr J, Trifunovic A, Larsson NG, Hoffer BJ, Olson L. High brain lactate is a hallmark of aging and caused by a shift in the lactate dehydrogenase A/B ratio. Proc Natl Acad Sci U S A 2010;107:20087-92.

37. Trapp GA. Plasma aluminum is bound to transferrin. Life Sci 1983;33:311-6.

38. Al-Hashem F. Camel's milk protects against aluminum chloride-induced normocytic normocromic anemia, lipid peroxidation and oxidative stress in erythrocytes of white albino rats. Am J Biochem Biotechnol 2009;5:126-36.

39. Goldstein ME, Cooper HS, Bruce J, Carden MJ, Lee VM, Schlaepfer WW. Phosphorylation of neurofilament proteins and chromatolysis following transection of rat sciatic nerve. J Neu- 
rosci 1987;7:1586-94.

40. Ekong MB, Ekpo MM, Akpanyung EO, Nwaokonko DU. Neuroprotective effect of Moringa oleifera leaf extract on aluminium-induced temporal cortical degeneration. Metab Brain Dis 2017;32:1437-47.
41. Khalil EA. Evaluation of the possible protective and therapeutic influence of coriander (Coriandum sativum L.) seed aqueous extract on hippocampal pyramidal cells against Alzheimer's disease induced by aluminum chloride in adult male albino rats. Researcher 2011;3:22-9. 